\title{
Glucocorticoid Signaling and Bone Biology
}

\author{
Author \\ Affiliation \\ T. Komori \\ Department of Cell Biology, Unit of Basic Medical Sciences, Nagasaki University Graduate School of Biomedical Sciences, \\ Nagasaki, Japan
}

\section{Key words \\ - apoptosis \\ - autophagy \\ wnt \\ osteoblast \\ - osteoclast \\ - OPG}

received 11.04.2016 accepted 08.06.2016

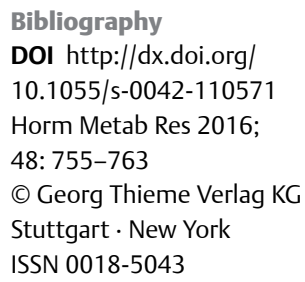

\section{Correspondence}

\section{T. Komori}

Department of Cell Biology Unit of Basic Medical Sciences Nagasaki University Graduate School of Biomedical Sciences Nagasaki 8528588

Japan

Tel.: + 81/95/849 7630

Fax:+81/95/849 7633

komorit@nagasaki-u.ac.jp

\section{Abstract \\ $\nabla$}

Since glucocorticoids remain an effective therapeutic option for the treatment of many inflammatory and autoimmune diseases, glucocorticoid-induced osteoporosis is the most common form of secondary osteoporosis. Fractures may occur in as many as $30-50 \%$ of patients receiving chronic glucocorticoid therapy. Under physiological conditions, glucocorticoids are required for normal bone development due to their regulation of osteoblast differentiation, possibly via the Wnt/ $\beta$-catenin pathway and TSC22D3. However, serum levels of endogenous corticosterone are elevated in aged mice and glucocorticoids exert negative effects on the survival of osteoblasts and osteocytes as well as angiogenesis. Glucocorticoid treatments impair bone formation and enhance bone resorption. Excess glucocorticoids induce osteoblast and osteocyte apoptosis by increasing pro-apoptotic molecules, reactive oxygen species, and endoplasmic reticulum stress and suppressing the $\mathrm{Wnt} / \beta$-catenin path-

\section{Introduction \\ $\nabla$}

Glucocorticoid (GC)-induced osteoporosis is the most common form of secondary osteoporosis. In humans treated with GCs, an early rapid decline in bone mineral density, possibly caused by enhanced bone resorption, is followed by a slower progressive decline in bone mineral density, which is due to reduced bone formation. Cancellous bone is preferentially lost in GCinduced osteoporosis, and fractures frequently occur at sites enriched in cancellous bone, such as the vertebrae and femoral neck [1]. Excess GCs are also associated with the early activation of genes associated with osteoclastogenesis and the later suppression of genes associated with osteogenesis and mineralization in mice [2]. A major way. Autophagy protects osteocytes from glucocorticoid-induced apoptosis, but passed some threshold, the process of autophagy leads the cells to apoptosis. Excess glucocorticoids impair osteoblastogenesis by inducing Wnt antagonists, including Dkk1, Sost, and sFRP-1. However, the findings are controversial and the involvement of Wnt antagonists requires further study. Excess glucocorticoids reduce the phosphorylation of Akt and GSK3 $\beta$, which enhances the degradation of $\beta$-catenin. Excess glucocorticoids have been shown to modulate the expression of miRNAs, including miR-29a, miR-34a-5p, and miR199a-5p, which regulate the proliferation and differentiation of osteoblast lineage cells. Excess glucocorticoids also enhance bone resorption by reducing OPG expression, increasing Rankl expression and reactive oxygen species, and prolonging the life span of osteoclasts; however, they also suppress the bone-degrading capacity of osteoclasts by disturbing the organization of the cytoskeleton.

issue in rodent models of GC-induced osteoporosis is that the loss of cancellous bone is not consistently observed in rodents. GC treatments in large animals, including rabbits and sheep, show a similar phenotype to that in humans [3]. GC receptor (GR, official symbol $\mathrm{Nr} 3 \mathrm{c} 1$ ) forms a complex containing chaperone molecules including HSP90 in the cytoplasm. Ligand binding to GR results in the dissociation of the complex and GR translocates into the nucleus. The GR homodimer directly binds GC responsive elements (GREs) in DNA and upregulates or suppresses the transcription of associated genes. Monomeric GR is also involved in the regulation of gene transcription. It binds to transcription factors by proteinprotein interactions and promotes or inhibits gene transcription [4]. 
The physiological functions of GCs on bone, the pathological effects of excess GCs on bone, and the pathogenesis of GCinduced osteoporosis in osteoblast and osteoclast lineages are described here.

\section{Physiological Functions of GCs in Bone}

The physiological functions of GR signaling in bone were examined using 2 methods ( $\bullet$ Fig. 1 ). In some tissues, GCs are locally metabolized before receptor binding by 2 enzymes: the inactivating enzyme 11ß-hydroxysteroid dehydrogenase type 2 (Hsd11b2), which converts biologically active GCs to their inert 11-keto metabolites, and the activating enzyme $11 \beta$-hydroxysteroid dehydrogenase type 1 (Hsd11b1), which works in the opposite manner. In order to inhibit the effects of GCs, human or rat HSD11B2 cDNA was overexpressed in various differentiation stages of osteoblast lineage cells or in osteoclasts.

In $\mathrm{Hsd11b2}$ transgenic mice under the control of the 2.3-kb rat Col1a1 promoter, which directs transgene expression to immature and mature osteoblasts, the trabecular bone volume in the lumber vertebrae in female mice and cortical thickness in both sexes are less than those in wild-type mice. Furthermore, osteoblast differentiation was found to be impaired in primary osteoblasts derived from Hsd11b2 transgenic mice [5,6] (॰ Fig. 1).
Another group also analyzed the same $H s d 11$ b2 transgenic line. Calvarial development was disturbed due to reduced bone formation, ectopic cartilage formation in the sagittal suture, and a defect in the postnatal removal of parietal cartilage ( $\bullet$ Fig. 1). These phenotypes were explained by a reduction in Wnt expression in osteoblasts, which leads to impaired osteoblast differentiation and a delay in cranial cartilage degradation by Mmp14, which is reduced in Hsd11b2 transgenic mice [7]. The same group showed significant reductions in the trabecular bone of the vertebrae and tibiae in $H s d 11 b 2$ transgenic mice of both sexes [8]. Similar phenotypes were also observed in $\mathrm{Hsd11b2}$ transgenic mice under the control of the $3.6-\mathrm{kb}$ rat Col1a1 promoter, which directs transgene expression in osteoblast precursors, immature osteoblasts, and mature osteoblasts [9].

GR-deficient $\left(\mathrm{GR}^{-/-}\right)$mice die just after birth due to atelectasis [10]. The skeletal development of $\mathrm{GR}^{-/-}$mice during the embryonic stage is similar to that of wild-type mice [11]. Since calvarial development is disturbed from the embryonic stage in Hsd11b2 transgenic mice under the control of the 2.3-kb Col1a1 promoter [7], ligand inactivation and GR deletion may cause different phenotypes. Osteoblast lineage-specific GR conditional knockout mice using Runx2 Cre transgenic mice show reductions in the trabecular bone mass, and the differentiation of primary osteoblasts from GR conditional knockout mice is impaired [11] ( $\odot$ Fig. 1). These findings indicate that GC is required for

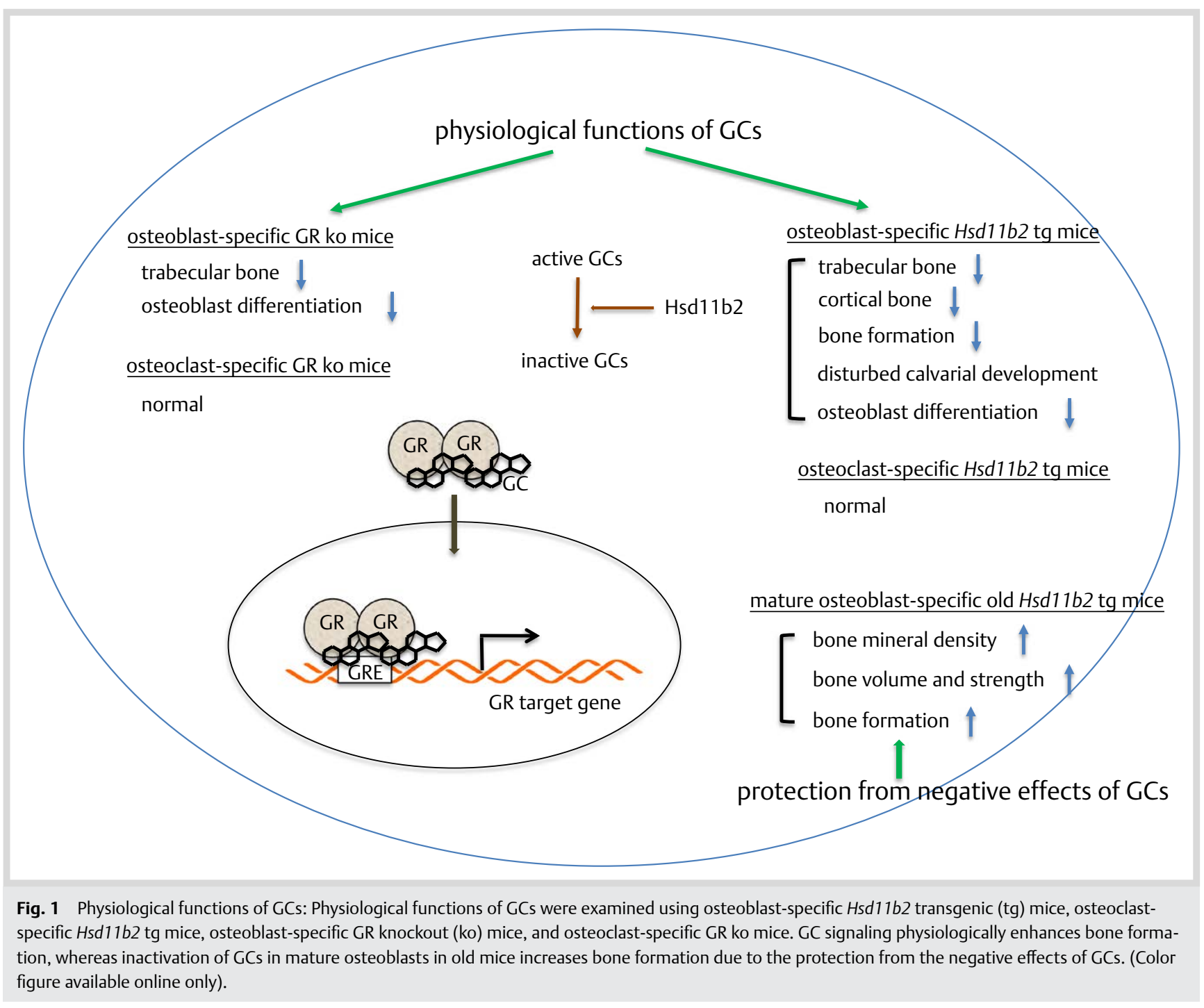


normal bone development and osteoblast differentiation under physiological conditions.

HSD11B2 transgenic mice under the control of the osteocalcin (Bglap2) promoter, which directs transgene expression in mature osteoblasts, show similar bone density, strength, and histomorphometric parameters to those in wild-type mice at 5 months of age [12]. The serum level of endogenous corticosterone is higher in 31-month-old wild-type mice than in 4-monthold wild-type mice, and bone mineral density, bone volume and strength, and bone formation are higher in HSD11B2 transgenic mice than in wild-type mice at 21 months of age. The increases observed in aged HSD11B2 transgenic mice appear to be attributed to the protection from the negative effects of GCs on the survival of osteoblasts and osteocytes as well as angiogenesis [13] ( $\bullet$ Fig. 1). Thus, GCs exert negative effects on the maintenance of bone in aged mice.
In $H s d 11 b 2$ transgenic mice under the control of the tartrateresistant acid phosphatase (Trap) promoter, which directs transgene expression to osteoclasts, bone mineral density and histomorphometric parameters were found to be similar to those in wild-type mice [14]. Furthermore, the osteoclast-lineage deletion of GR using Lyz2 Cre knock-in mice does not cause any abnormalities under physiological conditions [15]. Therefore, GC signaling has no significant effect on osteoclasts under physiological conditions.

Some reports suggested the mechanisms for the positive effects of GCs on osteoblasts. The overexpression of TSC22D3, also called the GC-induced leucine zipper (GILZ) that encodes a GC antiinflammatory effect mediator, under the control of the $3.6-\mathrm{kb}$ rat Col1a1 promoter increases bone mass due to enhanced bone formation. TSC22D3 interacts with C/EBPs and disrupts C/EBPmediated Ppary2 gene transcription [16]. Therefore, GCs may

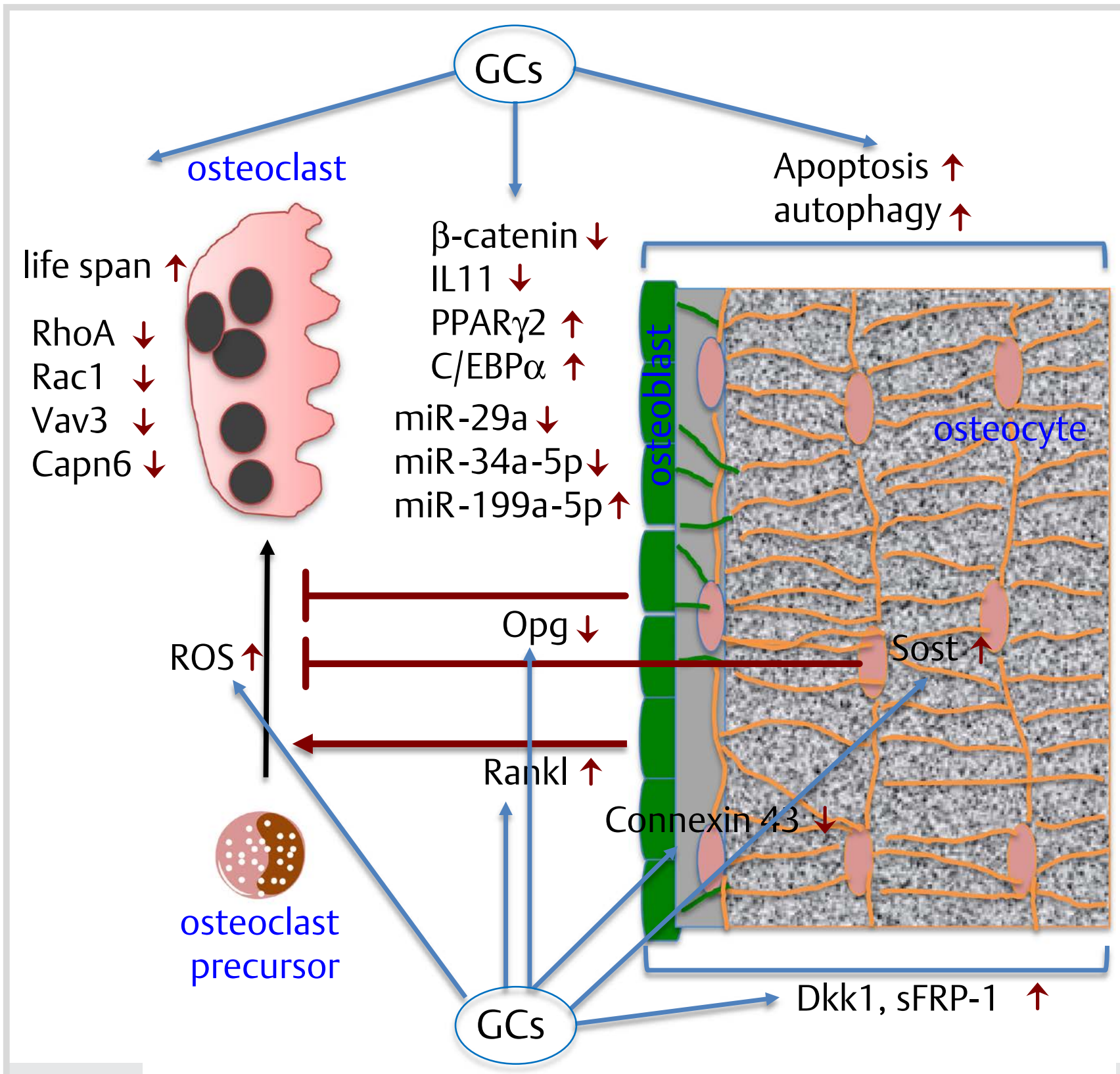

Fig. 2 Effects of excess GCs on osteoblasts, osteocytes, and osteoclasts: Excess GCs exert negative effects on osteoblast proliferation and differentiation as well as on the survival of osteoblasts and osteocytes. In contrast, excess GCs induce osteoclastogenesis and prolong the life span of osteoclasts, but inhibit their function. The key molecules involved in GC-induced osteoporosis are shown. (Color figure available online only). 
enhance osteoblast differentiation through TSC22D3, which inhibits mesenchymal stem cell differentiation into adipocytes. Dexamethasone (Dex) is used to enhance osteoblast differentiation in vitro; however, the underlying mechanisms have not yet been elucidated in detail. Dex was recently shown to stimulate the expression of fibronectin, integrin $\alpha 5$, and serum-GCinduced protein kinase 1 (SGK1), which induces Sec5-GEF-H1 interactions. Enhanced Sec5-GEF-H1 interactions promote GEF$\mathrm{H} 1$ to adhesion sites, which leads to the attachment of fibronectin to integrin at adhesion sites in order to generate cellular tension. These processes are required for the induction of osteoblast differentiation by Dex in vitro [17].

\section{Apoptosis Induced by Excess GCs}

$\nabla$

The administration of prednisolone $(2.1 \mathrm{mg} / \mathrm{kg} / \mathrm{d})$ for 27 days to mice has been shown to increase osteoblast apoptosis in the vertebrae by 3-fold and the frequency of osteocyte apoptosis in metaphysical cortical bone reached $28 \%$, resulting in a decrease in vertebral cancellous bone due to reduced bone formation [18]. Bisphosphonates and calcitonin prevent GC-induced apoptosis in osteoblasts and osteocytes [19]. The administration of GCs similarly reduces bone mass in wild-type mice and Bglap2promoter-HSD11B2 transgenic mice; however, the apoptosis of osteoblasts and osteocytes is induced in wild-type mice, but not in transgenic mice and vertebral compression strength is lost in GC-treated wild-type mice, but not in the transgenic mice [12] (० Fig. 2). Osteoblast apoptosis is prevented in $\mathrm{GR}^{-/-}$mice, but not in GR-dimerization-defective mice, indicating that GR dimerization is not required for osteoblast apoptosis induced by

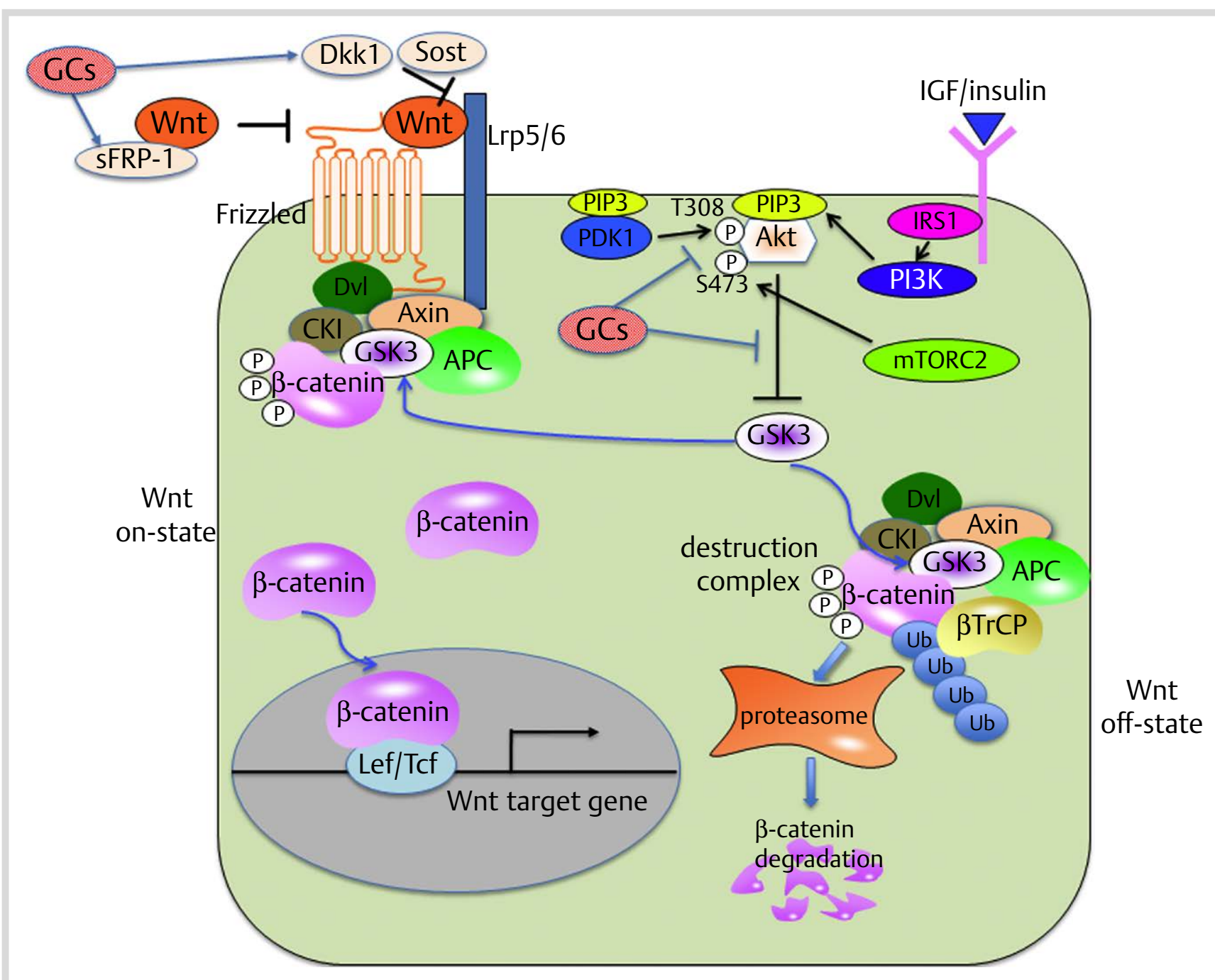

Fig. 3 Wnt signaling and PI3K-Akt signaling pathways: The binding of Wnt ligands to Fz and LRP5/6 leads to the membrane recruitment of the destruction complex, including Axin, APC, GSK3, CKI, and $\beta$-catenin, but not $\beta \operatorname{TrCP}$ by Dvl, which results in the inhibition of $\beta$-catenin ubiquitination and degradation. The destruction complex without $\beta \operatorname{TrCP}$ is saturated with phosphorylated $\beta$-catenin, and newly formed $\beta$-catenin freely translocates to the nucleus, binds to Lef/Tcf transcription factors, and induces the transcription of Wnt target genes. Dkk1 and Sost bind LRP5/6 and inhibit Fz-LRP5/6 complex formation. sFRP-1 binds to Wnts and inhibits binding to the receptors. In the absence of Wnt ligand binding, the destruction complex with $\beta \operatorname{TrCP}$ ubiquitinates $\beta$-catenin, which is then degraded in the proteasome. The destruction complex is recycled for $\beta$-catenin ubiquitination. Akt is phosphorylated by PDK1 and mTORC2, and phosphorylated (activated) Akt inhibits GSK3 by phosphorylation. Phosphorylated (inactivated) GSK3 loses the ability to phosphorylate $\beta$-catenin. Since the phosphorylation of $\beta$-catenin is required for $\beta$-catenin ubiquitination by $\beta \operatorname{TrCP}$, the degradation of $\beta$-catenin is inhibited. (Color figure available online only). 
GCs [11]. A number of mechanisms have been reported for GCinduced apoptosis. Excess GCs have been shown to increase the pro-apoptotic factors, Bim and Bak, and decrease the pro-survival factor, BclXL in vitro $[20,21]$. GCs induce the expression of E4bp4, which is a basic leucine zipper transcription factor, and upregulate Bim through E4bp4 [22]. Excess GCs also upregulate p53 protein levels, resulting in increases in p21, Noxa, and Puma [23]. Excess GCs activate Pyk2 by inducing $\mathrm{Ca}^{2+}$ entrance from the extracellular space, and the activated Pyk2 induces JNK activation followed by cell detachment and apoptosis [24]. Excess GCs increase reactive oxygen species (ROS), which induce apoptosis through the PKC $\beta /$ p66shc/JNK pathway, while excess GCs inhibit Akt, resulting in the suppression of the Wnt/B-catenin pathway, which is required for osteoblastogenesis and the prevention of cell death [25]. Ccl7, which is a chemotactic myokine, activates the $\beta$-catenin pathway through the phosphorylation of glycogen synthase kinase 3 (GSK-3), and exerts protective effects against the death of osteocyte-like MLO-Y4 cells induced by GCs [26]. Endoplasmic reticulum (ER) stress is alleviated by the phosphorylation of eIF2a, and inhibitors of eIF2a dephosphorylation have been shown to prevent GC-induced apoptosis in osteoblasts and osteocytes, indicating that the proapoptotic action of GCs is mediated, in part, through ER stress [27].

\section{Autophagy Induced by Excess GCs}

$\nabla$

A GC treatment increases the expression of the markers for autophagy in osteocytes ( $\bullet$ Fig. 2). When autophagy is inhibited, osteocyte numbers are decreased further by a GC treatment, suggesting that autophagy is a self-protective process in osteocytes in response to excess GCs [28]. Furthermore, the GC dosage determines osteocyte cell fate: a low dose of GCs induces autophagy while a high dose induces apoptosis [29]. Atg7 is involved in the formation of autophagosomes and is required for autophagy. Conditional Atg7 knockout mice in osteocytes using Dmp1 Cre transgenic mice show reduced osteoblast numbers and bone formation in cancellous bone; however, these are not reduced further by a GC treatment. Osteocyte density is also known to be reduced in conditional Atg7 knockout mice under physiological conditions, but is not reduced further by a treatment with GCs, indicating that the suppression of autophagy in osteocytes does not worsen the negative effects of GCs. These findings suggest that the process of autophagy is required for osteocyte death induced by GCs [30]. Therefore, autophagy appears to protect osteocytes from the negative effects of GCs; however, when these effects reach a certain threshold, the process of autophagy leads cells to apoptosis.

\section{The Role of Wnt Antagonists in GC-Induced Osteoporosis \\ $\nabla$}

The binding of Wnt ligands to their receptors frizzled (Fz) and LRP5 or LRP6 leads to the membrane recruitment of axin proteins by dishevelled (Dvl), which ultimately results in the inhibition of $\beta$-catenin ubiquitination and degradation ( $\odot$ Fig. 3). As a mechanism responsible for GC-induced reductions in bone formation, the modulated expression of Wnt antagonists has been extensively examined. Dkk1, which disrupts Wnt-induced FzLrp5/6 complex formation, is upregulated by Dex in primary cultured human osteoblasts, and Dex induces the reporter activity of the Dkk1 promoter [31,32]. Furthermore, the suppression of Dkk1 using antisense oligonucleotides or siRNA for Dkk1 abrogates the inhibition of osteoblast differentiation by GCs $[33,34]$. A Dex treatment in osteoblastic MC3T3-E1 cells induces the bone morphogenetic protein (BMP) antagonists follistatin and Dan, the Wnt antagonist, sFRP-1, and the Wnt signal inhibitor, Axin-2 [35] ( $\odot$ Fig. 3). However, GC-treated mice show variable results for the expression of Wnt antagonists. The expression of Dkk1 and Sost, which also disrupts Wnt-induced Fz-Lrp5/6 complex formation, is reduced by a prednisolone treatment for 7 days; however, after the treatment for 56 days, Dkk1 expression is increased while that of Sost returns to the level before the treatment [2]. Dkk1 expression is upregulated by a prednisolone treatment for 4 weeks [36]. A Dkk1 antisense treatment for 5 weeks was found to alleviate Dex-induced bone loss [33]. The treatment with prednisolone for 28 days increases Sost expression in wild-type mice at 5 months of age and reduces bone mass in wild-type mice but not in Sost $t^{-1-}$ mice. However, the reduction in bone formation and the increase in apoptosis are similarly observed between wild-type and Sost ${ }^{-1-}$ mice after the GC treatment [37]. In contrast, the protein expression of Dkk1 and Sost is reduced in bone marrow supernatants from wildtype mice after a GC treatment [30]. We also observed a reduction in Dkk1 and Sost mRNA levels in the osteocyte fraction in the mice at 12 weeks of age after a treatment with Dex for 4 weeks (unpublished observation). In humans, the expression of Dkk1 is decreased by short- and long-term treatments with GCs, while that of Sost only increases after the long-term treatment [38]. Therefore, the duration of GC treatment and the age of the animals may affect the response of Sost expression to excess GCs. Although Dkk1 is upregulated in human primary osteoblasts by a treatment with Dex, this upregulation is observed for $1-48 \mathrm{~h}[31,32]$. In a primary culture of GFP-positive osteoblasts from 2.3-kb Col1a1 promoter GFP transgenic mice, Dkk1 and sFRP- 1 are upregulated by a treatment with corticosterone for 1 and $0.5 \mathrm{~h}$, respectively, but return to control levels after $2 \mathrm{~h}$ [39]. These findings indicate that the upregulation of Dkk1 and sFRP-1 in vitro may be transient. Thus, the involvement of Dkk1 and other Wnt antagonists in the pathogenesis of GC-induced osteoporosis is still controversial and, thus, requires further study.

\section{Effects of Excess GCs through the Modulation of GSK3 $\beta$ \\ $\nabla$}

Dex reduces the phosphorylation of GSK3 $\beta$ and amount of nuclear $\beta$-catenin in MC3T3-E1 cells, and a treatment with the GSK3 inhibitor, 6-bromoindirubin-3'-oxime (BIO), mostly alleviates the negative effects of Dex in the bone of mice [40]. Furthermore, Dex inhibits Akt phosphorylation in mouse and human primary osteoblasts and osteoblastic MC3T3-E1 cells and also decreases $\beta$-catenin/TCF-dependent transcription in a GSK3dependent manner in MC3T3-E1 cells [41]. There are 2 glycogen synthase kinase 3 (GSK3) proteins, GSK3 $\alpha$ and GSK3 $\beta$. Three major pools of GSK3 exist under basal conditions: (1) GSK3 is part of the Wnt signaling complex; (2) GSK3 is part of the hedgehog signaling complex, which consists of Cos2, Gli, and other proteins; and (3) there is a free pool of GSK3. In Wnt signaling, GSK3 $\beta$ is a component of the destruction complex, which is composed of Axin, APC, CK1, and GSK3 $\beta$. When Fz-LRP5/6 receptors are not engaged, CK1 and GSK3 $\beta$ sequentially phosphorylate Axin-bound $\beta$-catenin. Phosphorylated $\beta$-catenin is recognized 
by $\beta$-TrCP, part of an E3 ubiquitin ligase complex, and the ubiquitinated $\beta$-catenin is rapidly degraded by proteasome. The IGFIGF receptor-PI3K-Akt pathway plays key roles in skeletal growth and endochondral ossification [42] ( $\odot$ Fig. 3). Akt phosphorylates Ser21 in GSK3 $\alpha$ and Ser9 in GSK3 $\beta$, which inactivates them. Contradictory findings have been reported for the involvement of Akt in Wnt signaling. Insulin and IGF have been shown to activate $\beta$-catenin/TCF-dependent transcription, mutant PI3K and kinase-dead Akt inhibited the $\beta$-catenin/TCF-dependent transcription induced by insulin and IGF, and constitutively active PI3K and Akt activated $\beta$-catenin/TCF-dependent transcription in a HepG2 hepatoma cell line [43]. Wnt was found to increase the phosphorylation of Akt; and phosphorylated (activated) Akt bound to the Axin-GSK3 $\beta$ complex in the presence of Dvl, phosphorylated GSK3 $\beta$, and increased free $\beta$-catenin in PC12 pheochromocytoma cells [44]. In contrast, Wnt failed to phosphorylate Akt, the introduction of kinase-dead GSK3 $\beta$ failed to activate $\beta$-catenin/TCF-dependent transcription in human embryonic kidney 293 cells, and insulin exhibited a limited ability to increase the Ser9 phosphorylation of Axin-2-bound GSK3 $\beta$ in 293 cells and CHOIR, a Chinese hamster ovary cell line stably expressing human insulin receptors [45]. Although these experiments were performed using similar methods, the findings obtained were contradictory. The activation of the Wnt pathway by PI3K-Akt signaling appears to be dependent on the cells used in the experiments. The physiological importance of the phosphorylation levels of GSK3 in Wnt signaling is also a matter of debate. In double homozygous knock-in mice in which GSK3 $\alpha$ (Ser21) and GSK3 $\beta$ (Ser9) were both changed to Ala, GSK3 $\alpha$ and GSK3 $\beta$ are constitutively active. However, $\beta$-catenin is increased and $\beta$-catenin/TCF-dependent transcription is activated under the stimulation of Wnt3a in GSK3 $\alpha /$ GSK3 $\beta$ homozygous knockin ES cells at similar levels to those in wild-type cells [46]. In a recently proposed model, the Wnt receptor-ligand interaction is shown to induce the dissociation of $\beta$-TrCP from the destruction complex, leaving phosphorylated $\beta$-catenin in the destruction complex, which is no longer ubiquitinated and degraded [47]. In this model, the destruction complex is saturated with phosphorylated $\beta$-catenin, and newly formed $\beta$-catenin freely translocates to the nucleus. In the absence of a Wnt stimulation, $\beta$-catenin is phosphorylated by CK1 and GSK3 $\beta$, phosphorylated $\beta$-catenin is ubiquitinated by $\beta-\operatorname{TrCP}$ in the destruction complex, and ubiquitinated $\beta$-catenin is degraded by the proteasome. The destruction complex is recycled for another round of $\beta$-catenin degradation ( $\odot$ Fig. 3). Therefore, the enhancement of GSK3 $\beta$ activity in this model is not a critical step in the inhibition of Wnt signaling as shown in GSK3 $\alpha /$ GSK3 $\beta$ homozygous knock-in ES cells $[46,47]$. Although excess GCs suppress bone formation, at least in part, through the activation of GSK3 $\beta$, the pathological importance of the effects of excess GCs in Wnt signaling through Akt requires further clarification.

A Dex treatment was previously shown to increase the expression of Dkk1 and PPAR 2 , reduce the phosphorylation of GSK3 $\beta$ and the amount of nuclear $\beta$-catenin, and induce adipocyte differentiation in the clonal mesenchymal progenitor cell line, ROB-C26 [48]. Furthermore, a Dex treatment increases the expression of C/EBP $\alpha$ by inhibiting the DNA hypermethylation of the promoter and induces adipocyte differentiation in bone marrow stromal cells, while the addition of $\mathrm{LiCl}$, which is an inhibitor of GSK3 $\beta$, alleviates the effects of Dex on the upregulation of C/EBP $\alpha$, promoter methylation, and the induction of adipocyte differentiation [49] ( $\odot$ Fig. 2). These findings indicate that
Dex induces adipocyte differentiation through the inhibition of Wnt signaling.

\section{MicroRNAs Involved in Impaired Osteoblastogenesis by Excess GCs \\ $\nabla$}

Several miRNAs, the expression of which is regulated by excess GCs, have been reported ( $\bullet$ Fig. 2). Excess GCs reduce the expression of miR-29a, and a treatment with miR-29a attenuates the adverse effects of GCs on bone by inducing the protein levels of Wnt3a, phosphorylated ERK, $\beta$-catenin, and Akt, and the mRNA levels of Runx2 and IGF-1, and by reducing the mRNA expression of Dkk1 and Rankl [50]. Furthermore, miR-29a has been shown to attenuate GC-mediated $\beta$-catenin deacetylation and ubiquitination, increase H3K9 acetylation on its own promoter, and enhance its own transcription by reducing the expression of HDAC4 [51]. GCs upregulate the expression of miR-34a-5p during bone marrow stromal cell proliferation, and miR-34a-5p reduces cell cycle-related proteins, including CDK4, CDK6, and Cyclin D1, leading to the inhibition of proliferation. In contrast, GCs reduce the expression of miR-34a-5p during osteogenic differentiation, leading to Notch signaling activation and the inhibition of osteogenic differentiation in bone marrow stromal cells [52]. Dex has been shown to upregulate the expression of miR199a-5p, which inhibits osteoblast proliferation by reducing FZD4 and Wnt2, and the deletion of miR-199a-5p attenuates Dex-inhibited osteoblast proliferation [53].

\section{Other Mechanisms Responsible for Impaired Bone Formation by GCs}

$\nabla$

GCs fail to reduce bone mass or bone formation in GR conditional knockout mice using Runx2 Cre transgenic mice. However, GC treatments have been shown to reduce bone mass and bone formation in mice carrying a mutation, which only disrupts GR dimerization, at similar levels to those in wild-type mice. These reductions are, in part, due to the inhibited expression of IL-11 and LIF by excess GCs in a manner that is independent of GR dimerization [11] ( $\odot$ Fig. 2). Dex reduces the expression of Connexin 43 through the Akt-mTOR pathway, and the overexpression of Connexin 43 attenuates Dex-inhibited cell viability and proliferation in MC3T3-E1 cells [54] ( $\odot$ Fig. 2). A previous study reported that GR interacts with Runx2 and inhibits the expression of Runx2 target genes [55]. Bone restitution during remodeling was recently found to require the recruitment of osteoprogenitors onto the reverse surface of the canopies, which are defined as a continuous layer of elongated cells lining the bone marrow and separated from the bone matrix by osteoclasts, reversal cells, or osteoblasts. A histomorphometric analysis of iliac crest biopsies from patients treated long-term with GCs revealed an increase in reversal surfaces, which lacked any neighboring osteoclasts or osteoids, and the coverage of the canopy [56]. Therefore, a decrease in the canopy is one of the characteristics of GC-induced osteoporosis. 


\section{Osteoclastogenesis Induced by Excess GCs}

\section{$\nabla$}

GCs enhance bone resorption in humans and mice $[2,57,58]$. Furthermore, a GC treatment increases osteoclastogenesis and bone resorption in vitro [59-61]. Rankl, which is expressed in stromal cells and osteoblast lineage cells, interacts with the cognate receptor Rank, which is expressed in osteoclast precursors and osteoclasts, resulting in osteoclastogenesis and the activation of osteoclasts. Osteoprotegerin (OPG), which is also expressed in stromal cells and osteoblast lineage cells, is a decoy receptor of Rankl and prevents the interaction of Rankl with Rank, thereby inhibiting osteoclastogenesis and osteoclast activation [62]. Enhanced bone resorption with a GC treatment has been explained by excess GCs severely reducing OPG mRNA expression and mildly increasing Rankl mRNA expression in stromal cells and osteoblastic cells, resulting in a marked increase in the RANKL/OPG ratio [60,63-65] ( $\bullet$ Fig. 2). Further, the reduction in OPG by excess GCs is abolished in Sost ${ }^{-1-}$ mice, indicating that Sost plays an important role in the regulation of OPG expression [37]. A GC treatment was previously found to increase the expression of Rankl, at least in part, through the suppression of miR-17/20a, which targets Rankl [66]. Although osteocytes strongly express OPG under physiological conditions, GC-induced osteocyte apoptosis further reduces OPG secretion to the bone surface and enhances osteoclastogenesis and osteoclast activation $[67,68]$ ( $\odot$ Fig. 2). Osteocyte apoptosis itself also enhances osteoclastogenesis, osteoclast activation, and bone resorption [68].

Excess GCs decrease the number of osteoclast progenitors, but increase the number of osteoclasts by prolonging their life span [69]. Furthermore, excess GCs decrease osteoclast numbers in $H s d 11 b 2$ transgenic mice under the control of the Trap promoter [14]. Therefore, excess GCs enhance bone resorption by extending the life span of osteoclasts. The autophagy of osteoclasts is also involved in GC-induced bone resorption because the pharmacological or genetic inactivation of autophagy ameliorates GC-induced bone loss by inhibiting osteoclastogenesis [70]. ROS are upregulated by excess GCs, and $N$-acetylcysteine (NAC), a ROS scavenger, has been shown to abrogate the effects of GCs on autophagy and osteoclastogenesis [61]. Therefore, ROS are involved in autophagy and GC-induced osteoclastogenesis (० Fig. 2).

A previous study reported that a GC treatment suppresses the bone-degrading capacity of osteoclasts by disturbing the organization of the cytoskeleton through the inhibition of the M-CSF activation of RhoA, Rac1, and Vav3 in wild-type osteoclasts, but not in osteoclast-specific GR-deficient osteoclasts in vitro [15] ( $\odot$ Fig. 2). Osteoclast-specific GR-deficient mice are protected from GC-suppressed bone formation, suggesting that GCs suppress bone formation via osteoclasts [15]. The overexpression of calpain 6 (Capn6), which is suppressed by Dex and involved in microtubule stability and acetylation as well as $\beta$-integrin expression, rescues the GC-mediated disruption of the osteoclast cytoskeleton [71] ( $\odot$ Fig. 2). As GC treatment in osteoclastogenesis in vitro increases or suppresses osteoclastogenesis and bone resorption under similar culture conditions, this discrepancy needs to be resolved. It has also been reported that GCs change the osteoclastic resorption mode. In the presence of excess GCs, osteoclasts elongate the excavations they initiated rather than migrating to a new resorption site, inducing deep resorption pits while reducing time-dependent increases in the resorption pit number [72].

\section{Conclusions}

$\nabla$

The GC pathway exerts positive effects on osteoblasts and bone formation under physiological conditions. However, excess GCs suppress bone formation by inducing apoptosis, inhibiting the Wnt signaling pathway, and modulating microRNA expression in osteoblasts and osteocytes. Excess GCs enhance bone resorption by suppressing OPG expression and prolonging the life span of osteoclasts ( $\bullet$ Fig. 2). Although many studies have focused on the role of the Wnt signaling pathway in the pathogenesis of impaired bone formation in excess GCs, the data obtained remain controversial. Furthermore, there are still a number of phenotypic differences in GC treatments between in vitro and in vivo experiments, among cells, among species, and between cancellous bone and cortical bone. Although many possible mechanisms for GC-induced osteoporosis have been reported, their pathological significance still needs to be evaluated.

\section{Acknowledgements}

$\nabla$

This work was supported by the grant from the Japanese Ministry of Education, Culture, Sports, Science and Technology to TK (Grant number: 26221310).

\section{Conflict of Interest \\ $\nabla$}

The author declares no conflict of interest.

\section{References}

1 Canalis E, Mazziotti G, Giustina A, Bilezikian JP. Glucocorticoid-induced osteoporosis: pathophysiology and therapy. Osteoporos Int 2007; 18: 1319-1328

2 Yao W, Cheng Z, Busse C, Pham A, Nakamura MC, Lane NE. Glucocorticoid excess in mice results in early activation of osteoclastogenesis and adipogenesis and prolonged suppression of osteogenesis: a longitudinal study of gene expression in bone tissue from glucocorticoidtreated mice. Arthritis Rheum 2008; 58: 1674-1686

3 Komori T. Animal models for osteoporosis. Eur J Pharmacol 2015; 759: 287-294

4 Kassel O, Herrlich P. Crosstalk between the glucocorticoid receptor and other transcription factors: molecular aspects. Mol Cell Endocrinol 2007; 275: 13-29

5 Sher LB, Woitge HW, Adams DJ, Gronowicz GA, Krozowski Z, Harrison JR, Kream BE. Transgenic expression of 11beta-hydroxysteroid dehydrogenase type 2 in osteoblasts reveals an anabolic role for endogenous glucocorticoids in bone. Endocrinology 2004; 145: 922-929

6 Sher LB, Harrison JR, Adams DJ, Kream BE. Impaired cortical bone acquisition and osteoblast differentiation in mice with osteoblasttargeted disruption of glucocorticoid signaling. Calcif Tissue Int 2006; 79: $118-125$

7 Zhou H, Mak W, Kalak R, Street J, Fong-Yee C, Zheng Y, Dunstan CR, Seibel MJ. Glucocorticoid-dependent Wnt signaling by mature osteoblasts is a key regulator of cranial skeletal development in mice. Development 2009; 136: 427-436

8 Kalak R, Zhou H, Street J, Day RE, Modzelewski JR, Spies CM, Liu PY, Li G, Dunstan CR, Seibel MJ. Endogenous glucocorticoid signalling in osteoblasts is necessary to maintain normal bone structure in mice. Bone 2009; 45: 61-67

9 Yang M, Trettel LB, Adams DJ, Harrison JR, Canalis E, Kream BE. Col3.6HSD2 transgenic mice: a glucocorticoid loss-of-function model spanning early and late osteoblast differentiation. Bone 2010; 47: 573-582

10 Cole TJ, Blendy JA, Monaghan AP, Krieglstein K, Schmid W, Aguzzi A, Fantuzzi G, Hummler E, Unsicker K, Schutz G. Targeted disruption of the glucocorticoid receptor gene blocks adrenergic chromaffin cell development and severely retards lung maturation. Genes Dev 1995; 9: $1608-1621$ 
11 Rauch A, Seitz S, Baschant $U$, Schilling AF, Illing A, Stride B, Kirilov M, Mandic V, Takacz A, Schmidt-Ullrich R, Ostermay S, Schinke T, Spanbroek $R$, Zaiss MM, Angel PE, Lerner UH, David JP, Reichardt HM, Amling M, Schutz G, Tuckermann JP. Glucocorticoids suppress bone formation by attenuating osteoblast differentiation via the monomeric glucocorticoid receptor. Cell Metab 2010; 11: 517-531

12 O'Brien CA, Jia D, Plotkin LI, Bellido T, Powers CC, Stewart SA, Manolagas SC, Weinstein RS. Glucocorticoids act directly on osteoblasts and osteocytes to induce their apoptosis and reduce bone formation and strength. Endocrinology 2004; 145: 1835-1841

13 Weinstein RS, Wan C, Liu Q Wang Y, Almeida M, O'Brien CA, Thostenson J, Roberson PK, Boskey AL, Clemens TL, Manolagas SC. Endogenous glucocorticoids decrease skeletal angiogenesis, vascularity, hydration, and strength in aged mice. Aging Cell 2010; 9: 147-161

14 Jia D, O'Brien CA, Stewart SA, Manolagas SC, Weinstein RS. Glucocorticoids act directly on osteoclasts to increase their life span and reduce bone density. Endocrinology 2006; 147: 5592-5599

$15 \mathrm{Kim}$ HJ, Zhao H, Kitaura H, Bhattacharyya S, Brewer JA, Muglia LJ, Ross FP, Teitelbaum SL. Glucocorticoids suppress bone formation via the osteoclast. J Clin Invest 2006; 116: 2152-2160

16 Pan G, Cao J, Yang N, Ding K, Fan C, Xiong WC, Hamrick M, Isales CM, Shi XM. Role of glucocorticoid-induced leucine zipper (GILZ) in bone acquisition. J Biol Chem 2014; 289: 19373-19382

17 Wang HL, Yang CH, Lee HH, Kuo JC, Hur SS, Chien S, Lee OK, Hung SC, Chang ZF. Dexamethasone-induced cellular tension requires a SGK1stimulated Sec5-GEF-H1 interaction. J Cell Sci 2015; 128: 3757-3768

18 Weinstein RS, Jilka RL, Parfitt AM, Manolagas SC. Inhibition of osteoblastogenesis and promotion of apoptosis of osteoblasts and osteocytes by glucocorticoids. Potential mechanisms of their deleterious effects on bone. J Clin Invest 1998; 102: 274-282

19 Plotkin LI, Weinstein RS, Parfitt AM, Roberson PK, Manolagas SC, Bellido T. Prevention of osteocyte and osteoblast apoptosis by bisphosphonates and calcitonin. J Clin Invest. 1999; 104: 1363-1374

20 Espina B, Liang M, Russell RG, Hulley PA. Regulation of bim in glucocorticoid-mediated osteoblast apoptosis. J Cell Physiol 2008; 215: 488-496

21 Chang JK, Li CJ, Liao HJ, Wang CK, Wang GJ, Ho ML. Anti-inflammatory drugs suppress proliferation and induce apoptosis through altering expressions of cell cycle regulators and pro-apoptotic factors in cultured human osteoblasts. Toxicology 2009; 258: 148-156

22 Chen F, Zhang L, OuYang Y, Guan H, Liu Q Ni B. Glucocorticoid induced osteoblast apoptosis by increasing E4BP4 expression via up-regulation of Bim. Calcif Tissue Int 2014; 94: 640-647

23 Li H, Qian W, Weng X, Wu Z, Zhuang Q Feng B, Bian Y. Glucocorticoid receptor and sequential P53 activation by dexamethasone mediates apoptosis and cell cycle arrest of osteoblastic MC3T3-E1 cells. PLoS One 2012; 7: e37030

24 Plotkin LI, Manolagas SC, Bellido T. Glucocorticoids induce osteocyte apoptosis by blocking focal adhesion kinase-mediated survival. Evidence for inside-out signaling leading to anoikis. J Biol Chem 2007; 282: $24120-24130$

25 Almeida M, Han L, Ambrogini E, Weinstein RS, Manolagas SC. Glucocorticoids and tumor necrosis factor alpha increase oxidative stress and suppress Wnt protein signaling in osteoblasts. J Biol Chem 2011; 286: 44326-44335

26 Kitase Y, Lee S, Gluhak-Heinrich J, Johnson ML, Harris SE, Bonewald LF. CCL7 is a protective factor secreted by mechanically loaded osteocytes. J Dent Res 2014; 93: 1108-1115

27 Sato AY, Tu X, McAndrews KA, Plotkin LI, Bellido T. Prevention of glucocorticoid induced-apoptosis of osteoblasts and osteocytes by protecting against endoplasmic reticulum (ER) stress in vitro and in vivo in female mice. Bone 2015; 73: 60-68

28 Xia X, Kar R, Gluhak-Heinrich J, Yao W, Lane NE, Bonewald LF, Biswas SK, Lo WK, Jiang JX.. Glucocorticoid-induced autophagy in osteocytes. J Bone Miner Res 2010; 25: 2479-2488

29 Jia J, Yao W, Guan M, Dai W, Shahnazari M, Kar R, Bonewald L, Jiang $J X$, Lane NE. Glucocorticoid dose determines osteocyte cell fate. FASEB J 2011; 25: 3366-3376

30 Piemontese $M$, Onal M, Xiong J, Wang Y, Almeida M, Thostenson JD, Weinstein RS, Manolagas SC, O'Brien CA. Suppression of autophagy in osteocytes does not modify the adverse effects of glucocorticoids on cortical bone. Bone 2015; 75: 18-26

31 Ohnaka K, Taniguchi H, Kawate H, Nawata H, Takayanagi R. Glucocorticoid enhances the expression of dickkopf- 1 in human osteoblasts: novel mechanism of glucocorticoid-induced osteoporosis. Biochem Biophys Res Commun 2004; 318: 259-264
32 Hurson CJ, Butler JS, Keating DT, Murray DW, Sadlier DM, O'Byrne JM, Doran PP. Gene expression analysis in human osteoblasts exposed to dexamethasone identifies altered developmental pathways as putative drivers of osteoporosis. BMC Musculoskelet Disord 2007; 8: 12

33 Wang FS, Ko JY, Yeh DW, Ke HC, Wu HL. Modulation of Dickkopf-1 attenuates glucocorticoid induction of osteoblast apoptosis, adipocytic differentiation, and bone mass loss. Endocrinology 2008; 149: 1793-1801

34 Butler JS, Queally JM, Devitt BM, Murray DW, Doran PP, O'Byrne JM. Silencing Dkk1 expression rescues dexamethasone-induced suppression of primary human osteoblast differentiation. BMC Musculoskelet Disord 2010; 11: 210

35 Hayashi K, Yamaguchi T, Yano S, Kanazawa I, Yamauchi M, Yamamoto $M$, Sugimoto T. BMP/Wnt antagonists are upregulated by dexamethasone in osteoblasts and reversed by alendronate and PTH: potential therapeutic targets for glucocorticoid-induced osteoporosis. Biochem Biophys Res Commun 2009; 379: 261-266

36 Thiele S, Ziegler N, Tsourdi E, De Bosscher K, Tuckermann JP, Hofbauer $L C$, Rauner M. Selective glucocorticoid receptor modulation maintains bone mineral density in mice. J Bone Miner Res 2012; 27: 2242-2250

37 Sato AY, Cregor M, Delgado-Calle J, Condon KW, Allen MR, Peacock M, Plotkin LI, Bellido T. Protection from Glucocorticoid-Induced Osteoporosis by Anti-Catabolic Signaling in the Absence of Sost/Sclerostin. J Bone Miner Res. 2016 doi:10.1002/jbmr.2869

38 Gifre L, Ruiz-Gaspa S, Monegal A, Nomdedeu B, Filella X, Guanabens N, Peris $P$. Effect of glucocorticoid treatment on Wnt signalling antagonists (sclerostin and Dkk-1) and their relationship with bone turnover. Bone 2013; 57: 272-276

39 Mak W, Shao X, Dunstan CR, Seibel MJ, Zhou H. Biphasic glucocorticoiddependent regulation of Wnt expression and its inhibitors in mature osteoblastic cells. Calcif Tissue Int 2009; 85: 538-545

40 Wang FS, Ko JY, Weng $L H$, Yeh DW, Ke HJ, Wu SL. Inhibition of glycogen synthase kinase-3beta attenuates glucocorticoid-induced bone loss. Life Sci 2009; 85: 685-692

41 Smith E, Frenkel B. Glucocorticoids inhibit the transcriptional activity of LEF/TCF in differentiating osteoblasts in a glycogen synthase kinase-3beta-dependent and -independent manner. J Biol Chem 2005; 280: 2388-2394

42 Rokutanda S, Fujita T, Kanatani N, Yoshida CA, Komori H, Liu W, Mizuno A, Komori T. Akt regulates skeletal development through GSK3, mTOR, and FoxOs. Dev Biol 2009; 328: 78-93

43 Desbois-Mouthon C, Cadoret A, Blivet-Van Eggelpoel MJ, Bertrand F, Cherqui G, Perret C, Capeau J. Insulin and IGF-1 stimulate the betacatenin pathway through two signalling cascades involving GSK-3beta inhibition and Ras activation. Oncogene 2001; 20: 252-259

44 Fukumoto S, Hsieh CM, Maemura K, Layne MD, Yet SF, Lee KH, Matsui T, Rosenzweig A, Taylor WG, Rubin JS, Perrella MA, Lee ME. Akt participation in the Wnt signaling pathway through Dishevelled. J Biol Chem 2001; 276: 17479-17483

45 Ding VW, Chen RH, McCormick F. Differential regulation of glycogen synthase kinase 3beta by insulin and Wnt signaling. J Biol Chem 2000; 275: 32475-32481

46 McManus EJ, Sakamoto K, Armit LJ, Ronaldson L, Shpiro N, Marquez $R$, Alessi DR. Role that phosphorylation of GSK3 plays in insulin and Wnt signalling defined by knockin analysis. EMBO J 2005; 24: 1571-1583

47 Li VS, Ng SS, Boersema PJ, Low TY, Karthaus WR, Gerlach JP, Mohammed $S$, Heck AJ, Maurice MM, Mahmoudi T, Clevers H. Wnt signaling through inhibition of beta-catenin degradation in an intact Axin1 complex. Cell 2012; 149: 1245-1256

48 Naito M, Omoteyama K, Mikami Y, Takahashi T, Takagi M. Inhibition of Wnt/beta-catenin signaling by dexamethasone promotes adipocyte differentiation in mesenchymal progenitor cells, ROB-C26. Histochem Cell Biol 2012; 138: 833-845

49 Li J, Zhang N, Huang X, Xu J, Fernandes JC, Dai K, Zhang X. Dexamethasone shifts bone marrow stromal cells from osteoblasts to adipocytes by C/EBPalpha promoter methylation. Cell Death Dis 2013; 4: e832

50 Wang FS, Chuang PC, Lin CL, Chen MW, Ke HJ, Chang YH, Chen YS, Wu SL, Ko JY. MicroRNA-29a protects against glucocorticoid-induced bone loss and fragility in rats by orchestrating bone acquisition and resorption. Arthritis Rheum 2013; 65: 1530-1540

51 Ko JY, Chuang PC, Chen MW, Ke HC, Wu SL, Chang YH, Chen YS, Wang FS. MicroRNA-29a ameliorates glucocorticoid-induced suppression of osteoblast differentiation by regulating beta-catenin acetylation. Bone 2013; 57: 468-475

52 Kang H, Chen H, Huang P, Qi J, Qian N, Deng L, Guo L. Glucocorticoids impair bone formation of bone marrow stromal stem cells by reciprocally regulating microRNA-34a-5p. Osteoporos Int 2016; 27: 1493-1505 
53 Shi C, Huang P, Kang H, Hu B, Qi J, Jiang M, Zhou H, Guo L, Deng L. Glucocorticoid inhibits cell proliferation in differentiating osteoblasts by microRNA-199a targeting of WNT signaling. J Mol Endocrinol 2015; 54: 325-337

54 Shen C, Kim MR, Noh JM, Kim SJ, Ka SO, Kim JH, Park BH, Park JH. Glucocorticoid Suppresses Connexin 43 Expression by Inhibiting the Akt/mTOR Signaling Pathway in Osteoblasts. Calcif Tissue Int 2016; 99: 88-97

55 Koromila T, Baniwal SK, Song YS, Martin A, Xiong J, Frenkel B. Glucocorticoids antagonize RUNX2 during osteoblast differentiation in cultures of ST2 pluripotent mesenchymal cells. J Cell Biochem 2014; 115: 27-33

56 Jensen PR, Andersen TL, Hauge EM, Bollerslev J, Delaisse JM. A joined role of canopy and reversal cells in bone remodeling - lessons from glucocorticoid-induced osteoporosis. Bone 2015; 73: 16-23

57 Dovio A, Perazzolo L, Osella G, Ventura M, Termine A, Milano E, Bertolotto A, Angeli A. Immediate fall of bone formation and transient increase of bone resorption in the course of high-dose, short-term glucocorticoid therapy in young patients with multiple sclerosis. J Clin Endocrinol Metab 2004; 89: 4923-4928

58 Brabnikova Maresova K, Pavelka K, Stepan JJ. Acute effects of glucocorticoids on serum markers of osteoclasts, osteoblasts, and osteocytes. Calcif Tissue Int 2013; 92: 354-361

59 Hirayama T, Sabokbar A, Athanasou NA. Effect of corticosteroids on human osteoclast formation and activity. J Endocrinol 2002; 175 : 155-163

60 Sivagurunathan S, Muir MM, Brennan TC, Seale JP, Mason RS. Influence of glucocorticoids on human osteoclast generation and activity. J Bone Miner Res 2005; 20: 390-398

61 Shi J, Wang L, Zhang H, Jie $Q$, Li X, Shi $Q$, Huang $Q$, Gao B, Han $Y$, Guo K, Liu J, Yang L, Luo Z. Glucocorticoids: Dose-related effects on osteoclast formation and function via reactive oxygen species and autophagy. Bone 2015; 79: 222-232
62 Suda T, Takahashi N, Udagawa N, Jimi E, Gillespie MT, Martin TJ. Modulation of osteoclast differentiation and function by the new members of the tumor necrosis factor receptor and ligand families. Endocr Rev 1999; 20: 345-357

63 Hofbauer LC, Gori F, Riggs BL, Lacey DL, Dunstan CR, Spelsberg TC, Khosla S. Stimulation of osteoprotegerin ligand and inhibition of osteoprotegerin production by glucocorticoids in human osteoblastic lineage cells: potential paracrine mechanisms of glucocorticoid-induced osteoporosis. Endocrinology 1999; 140: 4382-4389

64 Humphrey EL, Williams JH, Davie MW, Marshall MJ. Effects of dissociated glucocorticoids on OPG and RANKL in osteoblastic cells. Bone 2006; 38: 652-661

65 Kondo T, Kitazawa R, Yamaguchi A, Kitazawa S. Dexamethasone promotes osteoclastogenesis by inhibiting osteoprotegerin through multiple levels. J Cell Biochem 2008; 103: 335-345

66 Shi C, Qi J, Huang P, Jiang M, Zhou Q, Zhou H, Kang H, Qian N, Yang Q Guo L, Deng L. MicroRNA-17/20a inhibits glucocorticoid-induced osteoclast differentiation and function through targeting RANKL expression in osteoblast cells. Bone 2014; 68: 67-75

67 Moriishi T, Fukuyama R, Ito M, Miyazaki T, Maeno T, Kawai Y, Komori $H$, Komori T. Osteocyte network; a negative regulatory system for bone mass augmented by the induction of rankl in osteoblasts and sost in osteocytes at unloading. PLoS One 2012; 7: e40143

68 Komori T. Functions of the osteocyte network in the regulation of bone mass. Cell Tissue Res 2013; 352: 191-198

69 Weinstein RS, Chen JR, Powers CC, Stewart SA, Landes RD, Bellido T, Jilka RL, Parfitt AM, Manolagas SC. Promotion of osteoclast survival and antagonism of bisphosphonate-induced osteoclast apoptosis by glucocorticoids. J Clin Invest 2002; 109: 1041-1048

70 Lin NY, Chen CW, Kagwiria R, Liang R, Beyer C, Distler A, Luther J, Engelke $K$, Schett $G$, Distler JH. Inactivation of autophagy ameliorates glucocorticoid-induced and ovariectomy-induced bone loss. Ann Rheum Dis 2016; 75: 1203-1210

71 Hong JM, Teitelbaum SL, Kim TH, Ross FP, Kim SY, Kim HJ. Calpain-6, a target molecule of glucocorticoids, regulates osteoclastic bone resorption via cytoskeletal organization and microtubule acetylation. J Bone Miner Res 2011; 26: 657-665

72 Soe K, Delaisse JM. Glucocorticoids maintain human osteoclasts in the active mode of their resorption cycle. J Bone Miner Res 2010; 25: 2184-2192 